\title{
La Transición a la Agricultura y la Industrialización Cambiaron la Cara del Ser Humano. ¿Puede el Vegetarianismo ser un Nuevo Factor de Cambio?. Revisión de la Literatura
}

\author{
The Transition to Agriculture and Industrialization Changed the Human Face. \\ Can Vegetarianism be a New Factor of Change?. Review of the Literature
}

\author{
Natalia Morales $^{1}$ \& Viviana Toro-Ibacache ${ }^{1,2}$
}

MORALES, N. \& TORO-IBACACHE, V. La transición a la agricultura y la industrialización cambiaron la cara del ser humano. ¿Puede el vegetarianismo ser un nuevo factor de cambio? Revisión de la literatura. Int. J. Morphol., 36(1):35-40, 2018.

RESUMEN: Hace aproximadamente once mil años el ser humano pasó de alimentarse de los animales que cazaba o pescaba y los frutos y plantas que recolectaba, a aquellos que podía crecer a voluntad mediante la agricultura. Este cambio alteró dramáticamente la forma de la cabeza ósea y particularmente de la cara, que se hizo más grácil, y además se redujo el aporte de nutrientes claves. Con la industrialización, hubo un gran deterioro de la salud oral. Al abandono de una dieta cazadora recolectora incluso se le ha atribuido el origen de maloclusiones dentales, debido a la reducción del estrés masticatorio y con ello un menor tamaño relativo del maxilar y la mandíbula respecto a los dientes. Hoy en día, existiendo una mayor conciencia de la población respecto de sus cuidados y de su entorno, la adopción de dietas que excluyen cierto tipo de alimentos como los animales ha ganado adeptos. La dieta vegetariana presenta varias características que pueden afectar el metabolismo general y el óseo en particular, de manera similar a como lo hizo la agricultura en el pasado. La presente revisión busca analizar los cambios de la dieta humana, del punto de vista nutricional y mecánico y cómo estos afectaron la forma de la cara. Esto con el objetivo de comprender los posibles efectos de la introducción de alimentaciones de tipo restrictivas, como la vegetariana, en el organismo y particularmente en la anatomía facial.

PALABRAS CLAVE: Vegetarianismo; Masticación; Nutrición; Morfología craneofacial; Cambios en la alimentación.

\section{INTRODUCCIÓN}

La alimentación de un individuo es fundamental en su funcionamiento desde el punto de vista metabólico, económico, social, y también ecológico. En los animales, por ejemplo, cambios en la alimentación causados por fenómenos medioambientales son determinantes en la aparición de nuevas especies. El ser humano no es la excepción. La cabeza ósea es la parte del cuerpo donde se inicia el proceso de alimentación, y donde, como veremos más adelante, se pueden reflejar los mayores cambios fenotípicos derivados de cambios en él.

La dieta humana ha sufrido cambios radicales en su historia. Hasta el inicio del Holoceno, los individuos se alimentaron en base a lo que cazaban y recolectaban, para luego controlar el crecimiento y consumo de sus alimentos me- diante la agricultura, práctica aún fundamental en la vida del ser humano (Larsen, 2006).

La transición entre ambas economías de subsistencia implicó un cambio dramático en los nutrientes y características mecánicas de los alimentos. La agricultura colocó un énfasis mayor en las plantas, mientras que el alimento de origen animal disminuyó más o menos su importancia relativa. La industria introdujo un procesamiento mecánico y químico mayor de los alimentos, además de agregar elementos altamente cariogénicos como la azúcar refinada. Desde hace algunas décadas se han hecho más frecuente las dietas de tipo restrictivo, con las dietas vegetarianas como las más conocidas. El vegetarianismo consiste en la abstención del consumo de animales (parcial o total) y, en casos

\footnotetext{
${ }^{1}$ Centro de Análisis Cuantitativo en Antropología Dental e Instituto de Investigación en Ciencias Odontológicas, Facultad de Odontología, Universidad de Chile, Santiago, Chile.

${ }^{2}$ Departamento de Evolución Humana, Instituto Max Planck de Antropología Evolutiva, Alemania.

Este trabajo es financiado por la Comisión Nacional de Investigación Científica y Tecnológica de Chile (FONDECYT 11150175 a VT-I).
} 
más restrictivos, productos derivados de estos (huevos, leche, la miel y otros), por motivaciones religiosas, éticas, ambientalistas o culturales (Dinu et al., 2017).

El movimiento vegetariano, como se conoce actualmente, nace en 1847 encabezado por la Asociación Vegetariana Británica, el cual se apoya en la filosofía de antiguas religiones, como el budismo y el hinduismo, además de las enseñanzas del filósofo griego Pitágoras (Leitzmann, 2014). En la actualidad, hay pocos reportes del número de personas que eligen este tipo de dieta, pero de acuerdo a una investigación de Vegetarian Resource Group hecha en el 2006, en Estados Unidos habría de alrededor de 2,3 \% de la población que se considera vegetariana (Pribis et al., 2010). Si bien en Chile no hay estudios que describan qué porcentaje de la población sigue este tipo de alimentación, existen reportes que sugieren una proporción no menor de seguidores. El sitio web www.vegetarianoschile.cl publicó en 2017 los resultados de una encuesta en la que se consultaba a "más de 5.000 personas de todo Chile" acerca de distintos aspectos de políticas animalistas. En esta encuesta, del total de personas, un $34 \%$ se declaraban vegetarianos y un $13 \%$, veganos, mientras que la dieta predominante era la omnívora (Mac-Namara Barrenechea, 2017). La misma página publicó en 2013 un "censo vegetariano", el que fue contestado por 13.023 personas de las cuales un $81 \%$ se declaró vegetariana y un $19 \%$, vegana (Uribe, 2013). Hoy es común además encontrar tiendas de alimentos dedicados a la venta de productos vegetarianos y veganos. Interesantemente, si bien el vegetarianismo implica una serie de cambios en el modo de vida, el conocimiento de sus ventajas y desventajas a nivel del organismo no es necesariamente profundo por parte de quienes lo practican. Numerosas investigaciones concuerdan en que la dieta vegetariana presenta beneficios para la salud humana, entre ellos: el control de la hipertensión, disminución del riesgo de padecer enfermedades cardiovasculares, obesidad e incluso cáncer (Dinu et al.). Dentro de los problemas asociados se encuentra una reducción del consumo de proteínas, hierro y vitamina B12, lo que esto a una edad temprana lleva a alteraciones y retardo de crecimiento, anemia megaloblástica y problemas neurológicos respectivamente (Cayllante Cayllagua, 2014). También se puede dar una deficiencia de calcio, componente esencial del hueso, particularmente en dietas veganas (McEvoy \& Woodside, 2010). Brignardello et al. (2013) reportan un estudio en el que encuestó a 319 personas en Chile de entre 16 y 70 años autodeclaradas vegetarianas, con el objetivo de indagar en su grado de conocimiento acerca de la dieta que practican. De ellas, 266 personas se declararon vegetarianas y 53 veganos, y con un bajo conocimiento general acerca de las propiedades nutritivas de sus dietas. Estos resultados coinciden con Leitzmann quien reporta que, a nivel mundial, los vegetarianos tienden a desconocer cómo esta dieta podría afectarlos nutricionalmente de no existir una adecuada planificación. Cabe la interrogante de si este desconocimiento se extiende a todos los aspectos relativos a la alimentación: costo monetario y social, consecuencias metabólicas a largo plazo y cambios estructurales en el organismo. Es entonces importante generar la inquietud en la población y proveer de información que sirva de orientación a la comunidad.

El hueso es plástico, y su grado de plasticidad depende de su estructura microscópica y grado de mineralización, los que a su vez son dependientes del metabolismo, nutrición y cargas a las que son sometidos (Lieberman, 2011; Currey, 2012). El esqueleto facial no sólo recibe los nutrientes sistémicos sino que además es el lugar que se ve directamente afectado por cambios de las cargas masticatorias A nuestro conocimiento, sólo existe un artículo que evalúa el efecto de la dieta vegetariana en las características dentomaxilares de quienes la practican. En este estudio, adultos jóvenes vegetarianos presentaron un mayor grado de desgaste dentario oclusal, menor espacio disponible en la maxila y mandíbula para los dientes, y mayor grado de apiñamiento dentario en comparación a un grupo pareado control (Sherfudhin et al., 1996). Cabe entonces preguntarse si la adopción de una dieta vegetariana puede llevar a cambios metabólicos, mecánicos y por consecuencia, en la forma micro y macroscópica del hueso, en particular, de la forma facial. La presente revisión bibliográfica tiene como objetivo ahondar en esta interrogante.

Dieta y Evolución de la Forma Craneofacial. La forma de la cabeza ósea de distintas especies es capaz de reflejar su dieta más allá de las diferencias debidas a filogenia. En osos carnívoros que cambian su alimentación a una dieta basada en vegetales se ha observado una reducción del ancho craneal, dejando un menor espacio para los músculos elevadores de la mandíbula (Colangelo et al., 2012). Roedores que se alimentan con vegetales fibrosos muestran incisivos más anchos, filas de molares más largas y mandíbulas más robustas que en roedores con mayor diversidad de dieta, lo que se relacionaría con la necesidad de generar fuerzas oclusales más altas (Verde Arregoitia et al., 2017).

El consumo de carnes y particularmente de vegetales ha sido la base de la dieta del hombre moderno. Desde el punto de vista evolutivo, el consumo de nutrientes de origen animal ayudó al aumento del volumen cerebral, pero el cambio a una dieta basada en alimentos cultivados (arroz, maíz y otros) se asocia a malnutrición por falta de proteínas de la carne, disminución general de la robusticidad ósea debido al sedentarismo y un incremento en el número de caries (Larsen). El consumo de alimentos cultivados, de consistencia menos dura y tenaz, generó una reducción del tamaño facial por la reducción en el estrés masticatorio sobre 
los huesos de la cara (Lieberman). Con la industrialización, los alimentos altamente procesados, una alta presencia de caries y la pérdida prematura de dientes deciduos serían la causa de un menor tamaño de la maxila y la mandíbula, favoreciendo la aparición de maloclusiones (Corruccini, 1984). La pérdida prematura de dientes se asocia también a alteraciones de la forma de la articulación temporomandibular (Ballesteros Acuña et al., 2011). Los estudios de los cambios dietarios en distintas poblaciones sugieren que su impacto es tal, que sería la razón de la variación morfológica craneal alrededor del mundo (von Cramon-Taubadel, 2011). Sardi et al. (2006) comparó la morfología craneofacial de dos grupos humanos del centro - oeste de Argentina. El primer grupo, de 2.000 años de antigüedad tenía una economía de subsistencia de tipo cazador-recolector, con alimentos de origen principalmente animal y poco procesados. El segundo grupo, agricultores, procesaban más sus alimentos y su consumo de proteína animal era mucho menor. El grupo agricultor presentó un tamaño craneofacial menor, con una reducción de componentes masticatorios y de la fosa craneal posterior en comparación con el grupo cazadorrecolector, lo que ser relacionaría con un menor contenido nutricional y, especialmente, a una reducción de las fuerzas masticatorias relacionadas con el procesamiento de los alimentos (Sardi et al.). En un estudio análogo, Galland et al. (2016) comparó los cambios de la forma mandibular experimentados por una población de África en el transcurso de once mil años, durante los cuales la economía de subsistencia cambió de cazadora recolectora a agricultora avanzada. Los autores observaron una reducción significativa de la robusticidad mandibular en estos individuos. Por lo tanto, no sólo los nutrientes aportados por los alimentos, sino que también la consistencia de estos es un importante factor en la generación de cambios fenotípicos faciales en la población.

Desarrollo Facial, Alimentación y Función Masticatoria. El aparato masticatorio está formado por tejidos duros y blandos. El desarrollo pre y postnatal de los tejidos duros, y del esqueleto en general depende de la presencia de calcio y vitamina D (Mahon et al., 2010).

Los movimientos de la articulación témporomandibular son posibles gracias a la contracción de los músculos craneomandibulares y suprahioideos. Dentro de ellos, los involucrados en la generación de la fuerza de mordida durante la oclusión son el temporal, masetero y pterigoideo medial (ver detalles de sus características en Manns Freese, 2013).

Casi la totalidad de los huesos de faciales y de la calvaria se osifican de manera directa o endomembranosa, en el cual las células mesenquimáticas son transformadas en osteoblastos constituyendo un centro de osificación. La sínfisis (sincondrosis) y los procesos condilar y coronoide de la mandíbula se osifican a partir de cartílago secundario, el que experimenta una serie de cambios histológicos hasta formar un tejido óseo (Lieberman; Manns Freese). Una vez que el hueso está completamente modelado, su morfología cambia debido al proceso de remodelamiento en donde los osteocitos, al ser sensibles a la microdeformación ósea derivadas de las cargas mecánicas como la fuerza de mordida y la contracción muscular, pueden producir que los huesos cambien su forma para adaptarse a las fuerzas recibidas. De esta forma, si se ejerce una fuerza adicional prolongada, los huesos experimentarían cambios para mantener el óptimo funcionamiento mecánico de estos, es decir, para mantener una resistencia adecuada a las cargas, ocupando la menor cantidad de tejido posible (Lieberman; Currey).

Así, para que el desarrollo del esqueleto sea armónico, son fundamentales los nutrientes, pero también la acción muscular que le otorga las cargas necesarias para el modelamiento y remodelamiento, ya sea directamente a nivel de la inserción muscular o indirectamente mediante la acción mecánica generada. En el caso del esqueleto craneofacial, estas cargas indirectas provienen de la fuerza de mordida y la fuerza de reacción en la ATM. Estudios observacionales han demostrado la alteración del crecimiento óseo femoral en humanos cuyas madres presentaban insuficiencia de vitamina D (Mahon et al.). Experimentos en roedores han mostrado que la privación de actividad muscular altera la forma mandibular, particularmente de los procesos condilar, coronoides y angular, además de la forma del cráneo (Rot-Nikcevic et al., 2007). La reducción de la consistencia de la dieta también es un factor que altera la forma craneofacial en modelo murino, con gracilización craneomandibular (Spassov et al., 2017) y el acortamiento del arco maxilar (Beecher et al., 1981).

Masticación. La mecánica masticatoria se asocia a las partes de un ciclo, el cual se repite hasta que el alimento se pueda tragar, estas partes son:

- Apertura: la mandíbula desciende gracias a la fuerza de gravedad y a los músculos suprahioideos. Inicialmente las cabezas mandibulares giran alrededor del eje mediolateral. A esta rotación le sucede la contracción de los músculos pterigoídeos laterales, que traccionan el proceso condilar hacia adelante y abajo.

- Cierre: este se subdivide en dos etapas: el cierre rápido y el lento. El cierre rápido comienza con el trabajo del músculo pterigoídeo medial del lado de balance y el músculo masetero profundo del lado de trabajo, ambos elevan la mandíbula, la desplazan hacia posterior y ligeramente al lado de trabajo. El cierre rápido finaliza cuando los dientes pos- 
teriores mandibulares del lado de trabajo se ubican lateralizados con respecto a los dientes posteriores maxilares ipsilaterales. El cierre lento comienza con el movimiento hacia medial de los dientes posteriores mandibulares del lado de trabajo y el regreso de las cabezas mandibulares a la fosa mandibular. Este termina con el power stroke, porción crítica de la oclusión dental, en que los músculos maseteros, pterigoídeo medial y temporal, ejercen la máxima fuerza de contracción para triturar el alimento (Lieberman), y que por lo tanto tiene el mayor potencial de generar cambios anatómicos en el hueso.

Los dientes cumplen dos roles fundamentales: romper el alimento en base a la fuerza muscular, y transmitir las fuerzas oclusales al proceso alveolar maxilar o mandibular a través del ligamento periodontal. El resultado de este proceso también depende de las propiedades materiales de los alimentos.

Propiedades Mecánicas de los Alimentos y Fuerza Masticatoria. El primer paso en la fragmentación del alimento es su deformación inicial. Ésta se relaciona con el módulo de elasticidad del alimento, que es el grado de rigidez que posee un alimento de tal manera de que luego de ser deformado vuelva a su forma inicial tras liberarse la carga aplicada (Agrawal et al., 1998; Lieberman). El segundo ocurre cuando el alimento sufre deformación plástica: no puede volver a su forma original. Sobrepasada esta etapa, el alimento se rompe o fractura. Finalmente, la propagación de la fractura se va a ver dificultada o facilitada de acuerdo al grado de tenacidad del alimento (Lieberman).

Un alimento duro, como una manzana, zanahoria o una nuez, necesitan de una gran fuerza para generar la fractura, cuyo rasgo luego se propaga rápidamente. Por el contrario, un alimento tenaz necesita una gran cantidad de energía para propagar la fractura. La tenacidad de un alimento se debe normalmente a la presencia de colágeno (como en la carne) o de celulosa (como en las hojas de espinaca) (Lieberman). Tanto los alimentos duros como tenaces requieren de una mayor actividad muscular que los alimentos blandos (Agrawal et al.), pero la masticación de alimentos tenaces requiere un gran número de ciclos masticatorios y una actividad prolongada de los músculos elevadores mandibulares (Foster et al., 2006). Desde el punto de vista biomecánico, es este último tipo de carga el que tiene el mayor potencial de modificar la morfología ósea (Vickerton et al., 2014). Ahora bien, ¿qué propiedades mecánicas presentan alimentos de consumo común en una dieta omnívora?

Carnes. La carne roja cruda es elástica. Su dureza instrumental va de 18 a $69 \mathrm{~N}$ de fuerza requerida para la fractura del tejido, lo que no es alto, pero por ser tenaz requiere un alto número de ciclos masticatorios (de Huidobro et al., 2005) y con ello actividad muscular repetitiva para lograr fragmentarla. Esto se debe a que el tejido muscular de la carne es $75 \%$ agua y $20 \%$ proteína, de este último el $85 \%$ está constituido de por fibras musculares densamente compactadas, y fascículos recubiertos en tejido conectivo (Zink et al., 2014). Durante la cocción $\left(\right.$ a $60-90{ }^{\circ} \mathrm{C}$ ) el calor desnaturaliza y degrada las proteínas musculares, daña las membranas celulares y encoge las fibras musculares produciendo una disminución del volumen, elasticidad y tenacidad y un aumento de la dureza. Por lo tanto, cuando las carnes se cocinan cambian las propiedades materiales de la carne y con ello, la cantidad de ciclos de masticación necesarias para romperla (Zink et al.).

Vegetales. Un vegetal como la zanahoria cruda se caracteriza por su dureza, requiriendo de $190 \mathrm{~N}$ (Rastogi et al., 2008). La fractura de vegetales crudos se debe a la ruptura de las paredes celulares a causa de la tensión que genera la presión de turgencia interna de cada célula. Así, la mayor rigidez de los vegetales se da cuando se mantiene intacta la presión de turgencia interna de las células. Cuando los vegetales se cocinan por sobre $40^{\circ} \mathrm{C}$ las pectinas de sus paredes celulares se hidrolizan, aumentando la presión de turgencia, lo que conlleva a una disminución del módulo de rigidez y elasticidad, debido a que el agua invade los espacios intercelulares y rompe la adhesión celular. (Zink et al.).

Otros alimentos. La alimentación moderna implica no sólo la cocción sino además un alto grado de procesamiento mecánico de los alimentos. La trituración y corte por medio de instrumentos son capaces de transformar las propiedades materiales de cualquier alimento a uno que casi no requiera masticación. Tal es el caso de purés de frutas y verduras, carne molida, pescado desmenuzado, y alimentos de factura industrial como pastas, budines y otros. Otros productos ofrecen una mayor resistencia, como panes, galletas y confites. Sus características materiales dependen en gran parte de la forma de presentación del elemento y de cómo se mida su dureza, pero en general una papa frita requiere de $4 \mathrm{~N}$ de fuerza para su fractura, un suflé menos de $1 \mathrm{~N}$, y una galleta no esponjosa (tipo galleta de vino o de mantequilla), alrededor de $40 \mathrm{~N}$ (Vincent, 1998). La corteza del pan tipo Baguette requiere de aproximadamente $6 \mathrm{~N}$ (Gao et al., 2015). Estas propiedades son altamente variables, dependiendo de los ingredientes y métodos de cocción, los que son variados por la industria alimentaria de acuerdo a los requerimientos del mercado. En general, puede observarse que una característica común de la dieta moderna es que, si el alimento no es esencialmente blando, la dureza es una característica deseada por sobre la tenacidad, lo que es concordante con una disminución en la intensidad y frecuencia de la actividad masticatoria en los alimentos actuales. 


\section{DISCUSIÓN Y CONCLUSIÓN}

Los efectos de la alimentación sobre el organismo y el esqueleto en particular pueden clasificase latamente en dos tipos: aquellos derivados de su composición química y aquellos derivados de sus propiedades mecánicas. Las propiedades nutricionales son responsables no sólo del desarrollo de un individuo, sino que han sido un factor clave en los cambios fenotípicos evolutivos del ser humano (Larsen; Lieberman). Las propiedades mecánicas, por otra parte, se han asociado principalmente a cambios morfológicos de la cabeza ósea, tanto durante el desarrollo como en la evolución. Dietas en poblaciones arqueológicas consistentes en alimentos más duros y tenaces como semillas y carnes requieren una mayor fuerza oclusal o bien que ésta sea mantenida en el tiempo, generando mayor estrés y microdeformaciones en el cráneo y mandíbula. Estas cargas son esenciales en el crecimiento óseo, y se reconoce que el abandono de esta dieta, llevó el organismo a una serie de cambios anatómicos, además de las alteraciones nutricionales. Por otra parte, la dieta moderna urbana occidental es rica en alimentos altamente procesados fuera de la cavidad oral, los cuales presentan diversas propiedades nutricionales (o pueden no ser nutritivos), y su procesamiento intraoral produce menores fuerzas oclusales. Se debe tomar además en cuenta la pérdida de dientes debido a patologías infecciosas debido a una alimentación y/o higiene inadecuada, lo que limita la función masticatoria y altera la anatomía de componentes del sistema como la ATM. Cabe entonces preguntarse si las tendencias actuales hacia dietas que excluyen ciertos alimentos clave como la carne pueden inducir cambios morfológicos en la cara.

Como mencionamos anteriormente, la investigación respecto a los efectos sobre el organismo de la dieta vegetariana es amplia y llega a conclusiones similares: pueden existir deficiencias de nutrientes que, de no ser consideradas, pueden llevar a alteraciones metabólicas, entre ellas del hueso. Así, la adopción de una dieta vegetariana requiere de información y planificación por parte de quien la adopta, lo que como se mencionó, no siempre ocurre. Desde el punto de vista mecánico, los alimentos de una dieta vegetariana actual no difieren de mayor manera de una dieta omnívora, ya que la carne, si bien ofrece mayor resistencia mecánica que muchos otros alimentos, cocida de manera adecuada presenta una baja tenacidad. Las hojas, por ejemplo, de espinaca, son otro ejemplo de alimentos tenaces que cambian sus propiedades mediante la cocción, y están presentes tanto en dietas vegetarianas como omnívoras. Así, la cocción parece ser un factor fundamental en el análisis de las características mecánicas de los alimentos en cualquier tipo de dieta. Es interesante entonces traer a colación el caso de la dieta raw o crudívora. Esta nueva tendencia lentamente gana adeptos en occidente y consiste en comer principalmente alimentos crudos o cocidos a muy bajas temperaturas, aunque no necesariamente excluyendo carnes. Estas características la hacen de interés biomecánico, ya que podría considerarse a la dieta raw como un tipo de alimentación más ancestral, en el que se preservarían o se alterarían muy poco las propiedades materiales del alimento. Sin embargo, aunque pueden encontrarse en internet referencias a esta dieta desde hace varias décadas, muy pocos estudios se refieran a sus ventajas y desventajas nutricionales en humanos. A nivel facial, se ha visto que esta dieta está ligada a una mayor incidencia de erosiones dentales, las que serían de origen químico (frutas ácidas y uso de vinagres) pero que podrían llevar a un mayor desgaste dentario dada la disminución en la dureza del esmalte (Ganss et al., 1999), lo que podría causar alteraciones en la función masticatoria y consecuentemente, en la generación de fuerzas oclusales.

Además de las propiedades nutricionales y mecánicas de una dieta determinada, es importante tener en cuenta el momento del desarrollo óseo en que ésta es adoptada. No es lo mismo una dieta de bajo aporte nutricional y mecánico sobre un feto, un niño o un adulto. Un feto, a través de su madre, se ve afectado en etapas críticas de su crecimiento desde el punto nutricional, pero no mecánico (asumiendo que la deficiencia nutricional no afecta la actividad muscular fetal). Un niño se vería afectado nutricional y mecánicamente durante su desarrollo, y un adulto, en menor cuantía nutricionalmente y mucho menos, mecánicamente.

Para finalizar, debemos recalcar que el estudio de las consecuencias morfológicas de la función musculoesqueletal es un área de gran relevancia en medicina y odontología, tanto para el estudio de la etiología, prevención y tratamiento de alteraciones del sistema musculoesquelético. Además pero también en zoología, paleontología, antropología física y bioarqueología es de gran relevancia para conocer los factores subyacentes a los procesos evolutivos y de diversificación fenotípica, así como para reconstruir modos de vida de poblaciones extintas.

AGRADECIMIENTOS. A Dr. Adam van Casteren (Instituto Max Planck, Alemania).

MORALES, N. \& TORO-IBACACHE, V. The transition to agriculture and industrialization changed the human face. Can vegetarianism be a new factor of change? Review of the literature. Int. J. Morphol., 36(1):35-40, 2018.

SUMMARY: Approximately eleven thousand years ago humans beings went from feeding on animals they hunted or fished and fruits and plants they gathered, to crops they could grow through 
agriculture. This change dramatically altered the shape of the skull, particularly the face, which became more gracile, and also reduced the contribution of key nutrients. Along with industrialization, there was great deterioration of oral health. Leaving behind the hunter-gatherer diet has even been attributed to the origins of dental malocclusions, as masticatory stress was reduce, and reducing the size of the maxilla and mandible with respect to the teeth. Nowadays, there is greater awareness in the general population regarding personal care and their surroundings. Diets that exclude certain types of foods such as animal products are becoming more prevalent. The vegetarian diet has several characteristics that can affect metabolism, particularly the bones, as did the change from hunter-gatherer to agriculture in the past. The present review seeks to analyze the changes of the human diet, from the nutritional and mechanical point of view and how these have affected the shape of the face. This in order to understand the possible effects of the introduction of restrictive type feeds, such as the vegetarian diet in the body, particularly in facial anatomy.

KEY WORDS: Vegetarianism; Chewing; Nutrition; Craniofacial Shape; Dietary Changes.

\section{REFERENCIAS BIBLIOGRÁFICAS}

Agrawal, K. R.; Lucas, P. W.; Bruce, I. C., \& Prinz, J. F. Food properties that influence neuromuscular activity during human mastication. J. Dent. Res., 77(11):1931-8, 1998 .

Ballesteros Acuña, L. E.; Ramirez Aristeguieta, L. M. \& Muñoz Mantilla, G. Mandibular fossa depth variations: Relation to age and dental state. Int. J. Morphol., 29(4):1189-94, 2011.

Brignardello, G. J.; Heredia, P. L.; Paz Ocharán, S. M. P. \& Durán, A. S. Conocimientos alimentarios de vegetarianos y veganos chilenos. Rev. Chil. Nutr, 40(2):129-34, 2013

Cayllante Cayllagua, J. P. Vegetarianismo. Rev. Act. Clin. Med., 42:2195-9, 2014.

Colangelo, P.; Loy, A.; Huber, D.; Gomercic, T.; Taglianti, A. V. \& Ciucci, P. Cranial distinctiveness in the Apennine brown bear: genetic drift effect or ecophenotypic adaptation? Biol. J. Linn. Soc., 107(1):15-26, 2012.

Corruccini, R. S. An epidemiologic transition in dental occlusion in world populations. Am. J. Orthod., 86(5):419-26, 1984.

Currey, J. D. The structure and mechanics of bone. J. Mater. Sci., 47(1):41-54, 2012.

de Huidobro, F. R.; Miguel, E.; Blázquez, B. \& Onega, E. A comparison between two methods (Warner-Bratzler and texture profile analysis) for testing either raw meat or cooked meat. Meat Sci., 69(3):527-36, 2005.

Dinu, M.; Abbate, R.; Gensini, G. F.; Casini, A. \& Sofi, F. Vegetarian, vegan diets and multiple health outcomes: A systematic review with meta-analysis of observational studies. Crit. Rev. Food Sci. Nutr., 57(17):3640-9, 2017.

Foster, K. D.; Woda, A. \& Peyron, M. A. Effect of texture of plastic and elastic model foods on the parameters of mastication. J. Neurophysiol., 95(6):346979, 2006.

Galland, M.; Van Gerven, D. P.; Von Cramon-Taubadel, N., \& Pinhasi, R. 11,000 years of craniofacial and mandibular variation in Lower Nubia. Sci. Rep., 6:31040, 2016.

Ganss, C.; Schlechtriemen, M. \& Klimek, J. Dental erosions in subjects living on a raw food diet. Caries Res., 33(1):74-80, 1999.

Gao, J.; Wong, J. X.; Lim, J. C. S.; Henry, J. \& Zhou, W. Influence of bread structure on human oral processing. J. Food Eng., 167 Part B:147-55, 2015.

Larsen, C. S. The agricultural revolution as environmental catastrophe: Implications for health and lifestyle in the Holocene. Quat. Int., 150(1):1220, 2006.
Leitzmann, C. Vegetarian nutrition: past, present, future. Am. J. Clin. Nutr., 100(Suppl. 1):496S-502S, 2014.

Lieberman, D. E. The Evolution of the Human Head. Cambridge, Harvard University Press, 2011.

Mac-Namara Barrenechea, N. Chile: Resultados de Encuesta Política Animalista 2017. Santiago de Chile, Vegetarianos Chile, 2017. Disponible en: www.vegetarianoschile.cl/chile-resultados-de-encuesta-politica-animalista2017

Mahon, P.; Harvey, N.; Crozier, S.; Inskip, H.; Robinson, S.; Arden, N.; Swaminathan, R.; Cooper, C.; Godfrey, K. \& SWS Study Group. Low maternal vitamin D status and fetal bone development: cohort study. $J$. Bone Miner. Res., 25(1):14-9, 2010

Manns Freese, A. Sistema Estomatognático: Fundamentos Clínicos de Fisiología y Patología Funcional. Caracas, Amolca, 2013.

McEvoy, C., \& Woodside, J. V. Vegetarian and Vegan Diets: Weighing the Claims. In: Wilson, T.; Bray, G.; Temple, N. \& Struble, M. (Eds.). Nutrition Guide for Physicians. Nutrition and Health. Nueva York, Humana Press, 2010. pp.81-93.

Pribis, P.; Pencak, R. C. \& Grajales, T. Beliefs and attitudes toward vegetarian lifestyle across generations. Nutrients, 2(5):523-31, 2010.

Rastogi, N. K.; Nguyen, L. T. \& Balasubramaniam, V. M. Effect of pretreatments on carrot texture after thermal and pressure-assisted thermal processing. $J$. Food Eng., 88(4):541-7, 2008

Rot-Nikcevic, I.; Downing, K.; Hall, B. K. \& Kablar, B. Development of the mouse mandibles and clavicles in the absence of skeletal myogenesis. Histol. Histopathol., 22(1):51-60, 2007.

Sardi, M. L.; Novellino, P. S. \& Pucciarelli, H. M. Craniofacial morphology in the Argentine Center-West: consequences of the transition to food production. Am. J. Phys. Anthropol., 130(3):333-43, 2006.

Sherfudhin, H.; Abdullah, A.; Shaik, H. \& Johansson, A. Some aspects of dental health in young adult Indian vegetarians. A pilot study. Acta Odontol. Scand., 54(1):44-8, 1996.

Spassov, A.; Toro-Ibacache, V.; Krautwald, M.; Brinkmeier, H. \& Kupczik, K. Congenital muscle dystrophy and diet consistency affect mouse skull shape differently. J. Anat., 231(5):736-48, 2017.

Uribe, I. Chile: Resultados del Primer Censo Vegetariano. Santiago de Chile, Vegetarianos Chile, 2013. Disponible en: www.vegetarianoschile.cl/chileresultados-del-primer-censo-vegetariano

Verde Arregoitia, L. D.; Fisher, D. O. \& Schweizer, M. Morphology captures diet and locomotor types in rodents. R. Soc. Open Sci., 4(1):160957, 2017.

Vickerton, P.; Jarvis, J. C.; Gallagher, J. A.; Akhtar, R.; Sutherland, H. \& Jeffery, N. Morphological and histological adaptation of muscle and bone to loading induced by repetitive activation of muscle. Proc. Biol. Sci., 281(1788):20140786, 2014.

Vincent, J. F. V. The quantification of crispness. J. Sci. Food Agric., 78(2):1628,1998 .

von Cramon-Taubadel, N. Global human mandibular variation reflects differences in agricultural and hunter-gatherer subsistence strategies. Proc. Natl. Acad. Sci. U. S. A., 108(49):19546-51, 2011.

Zink, K. D.; Lieberman, D. E. \& Lucas, P. W. Food material properties and early hominin processing techniques. J. Hum. Evol., 77:155-66, 2014.

\section{Dirección para correspondencia: \\ Dra. Viviana Toro-Ibacache \\ Facultad de Odontología Universidad de Chile \\ Sergio Livingstone 943, Independencia \\ Región Metropolitana \\ CHILE}

Email: mtoroibacache@odontologia.uchile.cl

Recibido : 11-10-2017

Aceptado: 14-11-2017 